\title{
Duration of Hospital Stay Following Orthognathic Surgery at the Jordan University Hospital
}

\author{
Fadi Jarab $\cdot$ Esam Omar $\cdot$ Ahmed Bhayat • \\ Samir Mansuri $\cdot$ Sami Ahmed
}

Received: 20 July 2011/Accepted: 5 December 2011/Published online: 29 December 2011

(C) Association of Oral and Maxillofacial Surgeons of India 2011

\begin{abstract}
Background Major oral and maxillofacial surgery procedures have been routinely performed on an inpatient basis in order to manage both, the recovery from anesthesia and any unpredictable morbidity that may be associated with the surgery. The use of inpatient beds is extremely expensive and if the surgical procedures could be done on an outpatient setting, it would reduce the costs and the need for inpatient care. The aim was to determine the length of hospital stay (LHS) and the factors which influence the LHS following orthognathic surgery at the Jordan University Hospital over 5 years (2005-2009).

Methods This was a retrospective record review of patients who underwent orthognathic surgery at Jordan University Hospital between 2005 and 2009. The variables were recorded on a data capture form which was adapted and developed from previous studies. Descriptive and analytical statistical methods were used to correlate these variables to the LHS.

Results Ninety two patients were included in the study and $74 \%$ of them were females. The mean age was 23.7 years and the mean LHS was 4 days. The complexity of the procedure, length of operation time, intensive care unit (ICU) stay and year of operation were significantly correlated with a positive LHS $(P<0.05)$.

Conclusion Patients' hospital stay was directly related to the complexity of the orthognathic procedure, the operation time, time spent in ICU and the year in which the operation was done. There was a significant reduction in the LHS over the progressing years and this could be due to an
\end{abstract}

F. Jarab · E. Omar · A. Bhayat $(\bowtie) \cdot S$. Mansuri - S. Ahmed College of Dentistry, Taibah University, Al-Madinah, Kingdom of Saudi Arabia

e-mail: ahmedbhayat6@gmail.com increase in experience and knowledge of the operators and an improvement in the hospital facilities.

Keywords Orthagnathic surgery $\cdot$ Length of hospital stay $\cdot$ Factors responsible for length of stay

\section{Introduction}

Jordan University Hospital (JUH) is a tertiary educational center with a residency program for oral and maxillofacial surgery. It offers residents the opportunity to specialize in oral and maxillofacial surgery which includes orthognathic surgery. Orthognathic surgery is a versatile, widely accepted procedure for the correction of dentofacial deformities. The benefits of orthognathic surgery are well-documented and include three main aspects: improved dental and facial aesthetics, better dental function, and improvements in psychosocial characteristics and quality of life [1-3].

Major oral and maxillofacial surgery, including orthognathic surgery, has traditionally been performed on an inpatient basis. The rationale for inpatient care was based on the need to manage the recovery from anesthesia, potential airway instability, homeostasis, resumption of oral intake, pain control, and any unpredictable morbidity associated with maxillofacial surgical cases [4]. Reports from the United Kingdom have shown that the use of inpatient beds following surgery have been 'the most expensive resource of the National Health Services' [5]. This high cost could be one of many reasons for the increase in day care surgeries. Other reasons could include patient preference which would result in reduced cancellation of lists and value-for-money outcomes [6].

There is scant information concerning the needed in patient stay after orthognathic surgery and the factors that 
affect that stay. The transition of oral and maxillofacial surgical care to an outpatient setting challenges preconceptions regarding the morbidity associated with these procedures [7]. Many authors have reported that if oral and maxillofacial surgical care could be provided with less morbidity and rapid postoperative recovery, the inpatient care will not be necessary, the patient experience with the surgery will be better and the cost would be reduced [8-10].

The LHS is reported to range from 1.3 to 8 days $[8,9]$. Many factors have been shown to be associated with the LHS but most of the authors agree that the LHS is influenced by the anesthesia time, the operation time and the complexity of the operation [7-9].

The aim was to determine the LHS following orthognathic surgery and the factors that influence the length of stay.

\section{Methods}

This was a retrospective record based study and included all patients who attended the JUH for orthognathic surgery between January 2005 and December 2009 (5 years). A total of 92 records who met the criteria were included in the study. For inclusion in the study, patients were classified as "healthy" according to the criteria of the American Society of Anesthesiologist (ASA I). In addition, only patients who underwent internal fixation using mini plates were included in this study. A data collection form was developed from previous studies [5] and modified in order to collect the necessary information. All of the data was obtained from the patients' record chart and if data was missing or incomprehensible, the patient was excluded from the study. There were five variables and each variable was further subdivided into two or more sub-variables. The data collection form consisted of both open and closed ended questions and included the following:

1. demographic data which included the age and gender of the patient

2. the type of operative care which was classified into the following groups:

The type of malocclusion present and the type and complexity of the surgical procedure which was coded from 1 to 4 as described below:

a) genioplasty

b) single jaw osteotomy (Lefort I, or Sagittal Split osteotomy)

c) two jaw surgery (bimaxillary osteotomy)

d) combined procedures that included any of the above procedures done with ancillary procedures as augmentation with bone graft, septoplasty, zygomatic osteotomy or tempro-mandibular joint TMJ surgery
3. the duration of the surgery which was calculated in hours from the time the patient was anesthetized up to the time he/she was sent to the recovery room

4. the year in which the surgical procedure was performed and ranged from 2005 till 2009

5. The post operative care which was coded as either " 0 " (no life threatening complications) or "1" (presence of complications). These complications included: admittance and stay in the intensive care unit (ICU) and life threatening complications such as:

a) airway management (requiring intubation after recovery) and/or

b) excessive bleeding that required blood transfusion

The outcome variable, the total length of hospital stay in days, was extracted from admission and discharge notes.

Data analysis was completed using Statistical Package for Social Science (SPSS) version 15. Descriptive analysis for each factor was computed, bivariate analysis was then done for all of these factors in relation to the LHS. Those significant factors found in the bivariate correlation were then assessed using multiple regression analysis. This was used to confirm the significance of the correlation with the LHS $(P<0.05)$.

Ethics was obtained for the study from the Jordan University Hospital Ethics Committee.

\section{Results}

A total of 92 patients met the criteria and were included in the study. The mean age was $23.7(\mathrm{SD} \pm 4.7)$ years and ranged between 16 and 44 years. The female patients constituted $74 \%$ of the total with a female to male ratio of 2.7:1. The prevalence of the different types of malocclusion is shown in Fig. 1. The most common types were skeletal class $3(35 \%)$ and facial asymmetry (21\%).

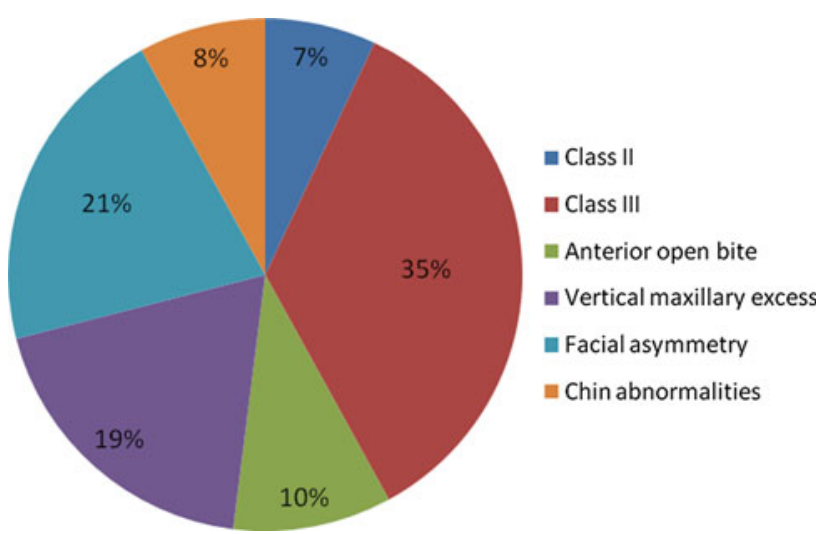

Fig. 1 The classification of the different types of Malocclusions $(N=92)$ 
The different types of surgical procedures that were performed included: Genioplasty with all its modifications 17\%; single jaw surgery [Le Fort I or Bilateral Sagittal Split osteotomy] 24\%; two jaw surgery [bimaxillary osteotomy] $42 \%$; and combined procedures constituted $18 \%$. The most common types were two jaw surgery $(42 \%)$ and single jaw surgery (24\%) which included either Lefort I (8 cases) or Bilateral Sagittal Split osteotomy (16 cases). There were no combined procedures that included ancillary procedures as augmentation with bone graft, septoplasty, zygomatic osteotomy or TMJ surgery.

The mean operation time was $4 \mathrm{~h}(\mathrm{SD} \pm 1.6 \mathrm{~h})$ and ranged from 1 to $8 \mathrm{~h}$. The operation time was significantly correlated to the complexity of the procedure $(P<0.01)$ and increased the LHS $(P<0.05)$.

The duration of stay in the ICU varied as shown in Fig. 2. Almost two-thirds (64\%) did not require staying in the ICU and were transferred to the general ward on the same day that the procedure was performed. The length of ICU stay directly increased the LHS $(P<0.05)$.

A total of 10 cases (13\%) experienced a life threatening complication (code 1). This included airway management and intubation (4 cases) and blood transfusions as a result of excessive bleeding ( 6 cases). All patients who presented with either or both of the two life threatening complications spent more time in the ICU. There was a positive correlation $(P<0.01)$ between the presence of a life threatening complication and the length of stay in the ICU.

The mean LHS was 4.2 days with a mean of 4 days and a range between 0 and 9 days. The average LHS decreased over the 5 year study period from 5 days in 2005 to 3.7 days in 2009. There was a statistical correlation $(P<0.01)$ between the average LHS and the year in which the procedure was performed. Those performed in 2007, 2008 and 2009 were likely to have a shorter LHS compared to patients who were treated in 2005 and 2006. There was an increase in the total number of orthognathic surgical

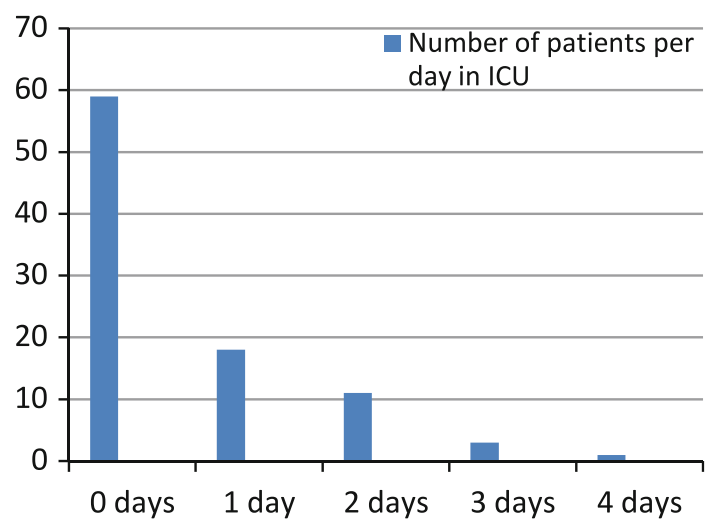

Fig. 2 The number of days each patient spent in ICU following surgery $(N=92)$ procedures performed from 6 cases in 2005 to 52 cases in 2009.

By using bivariate analysis (Spearman correlation) 4 factors were found to be significantly correlated with LHS $(P<0.05)$; these were

1. the type of surgical operation that was performed

2. the duration (in hours) of the surgical procedure

3. the number of days spent in the ICU after surgery

4. the year in which the surgery was performed

The other factors such as age and gender were not significantly correlated to the length of hospital stay and were therefore not included in any further statistical analyses.

\section{Discussion}

There was no statistical relationship between the age and the gender of patients to the LHS; this was also reported by other authors $[7,10]$. The average LHS was 4.2 days which lies within the reported range of between 1.3 to 8.5 days [11-13]. Over the 5 year study period there was a significant reduction in the LHS from 5 to 3.7 days $(P<0.01)$ even though there was an increase in the number of operations done from 6 cases in 2004 to 52 cases in 2009. The increase in the number of cases during the study period could be attributed to an increase in awareness and education amongst the general public in relation to their facial esthetics. Over the study period, the population was increasingly exposed to international television and media which could have increased their need for facial improvement. It is also possible that over the study period, the clinical and support staff improved their techniques and clinical skills. This could have reduced some of the complications and hence reduced the LHS. Lastly, the reduction in LHS could be due to an increase in the number and type of surgical instruments which impacted on anesthetic time and indirectly on LHS.

Many authors $[4,7,10]$ have reported that the length of anesthesia time as a significant predictor of the need for subsequent hospitalization. According to Lupiro [7] there was a positive correlation between the duration of the procedure (including anesthesia time) and the admission for observation. All of the surgical procedures which lasted more than $4 \mathrm{~h}$ and $28 \mathrm{~min}$ were significantly associated with admission for observation. Dann [4] concluded that both the length of time under anesthesia and the duration of the surgical procedure strongly correlated with postoperative morbidity, surgical outcomes, and the need for subsequent inpatient surgical care. The mean operation time in the current study was $4 \mathrm{~h}$ and this was measured from anesthesia time till full recovery of the patient. This was slightly higher than other published studies $[4,7,10]$ and 
could be due to a lack of appropriately trained nurses, a shortage of instruments and a lack of operator experience during the initial years in which the study was conducted.

ICU stay in the past was common for patients who had undergone orthognathic surgical procedures and depended on the need for airway management or other supportive care [14]. Potential sources of immediate postoperative airway compromise after orthognathic surgical procedures included edema due to excessive and or prolonged retraction of tissues medial to the ascending ramus of the mandible, extensive and or prolonged tissue dissection, supraperiosteal tissue dissection, and excessive manipulation of dental-osseous segments and excessive bleeding with subsequent hematoma formation in tissue spaces that impinge upon the airway, such as the nasopharynx, oropharynx, hypopharynx, and sublingual space. The situation is further complicated by postoperative trismus or limited mouth opening and the possibility of intermaxillary fixation or wiring of the jaws [8].

Upper airway obstruction following orthognathic surgery is a rare but potentially life-threatening complication, there have been anecdotal reports of upper airway obstruction following orthognathic surgery, but the exact incidence is uncertain. A review of the literature from the past 15 years failed to provide an incidence of postoperative complications related to upper airway compromise [8]. An abstract on mortality associated with orthognathic surgery in Canada reported that of the four hundred sixty-six cases, only 1 case was associated with orthognathic surgery [9]. For this reason, some institutions have strict criteria for extubation, requiring that all orthognathic surgery patients be admitted to an ICU or that they remain intubated overnight following surgery. Other centers advocate early extubation and have apparently not reported any significant adverse events $[15,16]$. Other than airway management, there was a concern about increased blood loss with Le Fort I procedures both intraoperatively and delayed bleeding [13]. However, authors do agree [11] that autodonations or transfusions are not necessary for single-jaw surgery and not required in all cases of bimaxillary osteotomy unless an iliac crest graft is included in the procedure. Therefore, in the absence of postoperative bleeding, progressive swelling, or co morbidities that mandate continuous physiologic monitoring, it does not appear that routine admission of patients to an ICU or overnight intubation postoperatively is necessary. However, there can be no substitute for clinical judgment that is performed by the anesthetist who provided the post operative care [8].

In the JUH, a bed in the ICU was reserved for all patients planned to have two jaw surgery (bimaxillary osteotomy) the night before, each case was assessed individually and the admission to the ICU was decided in cooperation between the surgeon and the anesthetist.
Lombardo et al. [12] and Dolan and white [17] noted a procedure-based LHS pattern, reporting the longest LHS in bimaxillary procedures, followed by maxillary procedures and then mandibular procedures. Lupori [7] determined that increased duration of anesthesia and increased number of procedures resulted in increased frequency of hospital admissions. Interestingly, these authors found that the use of ancillary procedures increased LHS, but the increase was not statistically significant, and there was no significant difference between Le Fort I and Bilateral Sagittal Split osteotomy in relation to LHS. In this study, a significant correlation was observed with increasing complexity of the procedure and the LHS $(P=0.009)$, and no significant difference between the single jaw operations such as Le fort I or Bilateral Sagittal Split Osteotomy and the length of stay for any one of them.

\section{Conclusion}

The patients' hospital stay was directly related to the complexity of the orthognathic procedure, the operation time, the length of time spent in the ICU and the year in which the operation was done. A significant reduction was noticed in LHS over the progressing years and this may reflect an increase in experience and knowledge amongst the clinicians and an improvement in the medical facilities.

\section{References}

1. Hunt NP, Cunningham SJ (1997) The influence of orthognathic surgery on occlusal force in patients with vertical facial deformities. Int J Oral Maxillofac Surg 26:87-91

2. Proffit WR, Turvey TA, Fields HW, Phillips C (1989) The effect of orthognathic surgery on occlusal force. J Oral Maxillofac Surg 47:457-463

3. Cunningham SJ, Hunt NP, Feinmann C (1995) Psychological aspects of orthognathic surgery: a review of the literature. Int $\mathrm{J}$ Adult Orthod Orthognath Surg 10:159-172

4. Dann J (1998) Outpatient oral and maxillofacial surgery: transition to a Surgicenter Setting and Outcome of the first 200 cases. J Oral Maxillofac Surg 56:572-577

5. Black D, Pearson M (2002) Average length of stay, delayed discharge and hospital congestion. Br Med J 325:610-611

6. Audit commission (1991) measuring quality: the patient's view of day surgery. Occasional paper

7. Huamán ET, Juvet LM, Nastri A, Denman WT, Kaban LB, Dodson TB (2008) Changing patterns of hospital length of stay after orthognathic surgery. J Oral Maxillofac Surg 66(3):492

8. Meisami T, Musa M, Keller MA, Cooper R, Clokie CM, Sàndor GK (2007) Magnetic resonance imaging assessment of airway status after orthognathic surgery. Oral Surg Oral Med Oral Pathol Oral Radol Endod 103:458-463

9. Parbatani R, Williams AC, Ireland AJ, Sandy JR (2009) The process of orthognathic care in an NHS region. Ann R Coll Surg Engl 92(1):34-39 
10. Garg M, Cascarini L, Coombes DM, Walsh S, Tsarouchi D, Bentley R, Brennan PA, Dhariwal DK (2010) Multicentre study of operating time and inpatient stay for orthognathic surgery. Br J Oral Maxillofac Surg 48(5):360-363

11. Samman N, Cheung LK, Tong AC, Tideman H (1996) Blood loss and transfusion requirements in orthognathic surgery. $\mathrm{J}$ Oral Maxillofac Surg 54:21-26

12. Lombardo GA, Karakourtis MH, White RP Jr (1994) The impact of clinical practice patterns on hospital charges for orthognathic surgery. Int J Adult Orthod Orthogn Surg 9:251-258

13. Solomons NB, Blumgart R (1988) Severe late onset epistaxis following Lefort I osteotomy: angiographic localization and embolization. J Laryngol Otol 102:206-213
14. George H, Blakey H, Raymond P (1999) Bilateral sagittal split osteotomies in an ambulatory care setting. Semin Orthod 5:241-243

15. Zulian MA, Chisum JW, Mosby EL, Hait WR (1989) Extubation criteria for oral and maxillofacial surgery patient. J Oral Maxillofac Surg 47:616-620

16. Haber-Cohen A, Rothman M (1988) A survey of extubation practices following orthognathic surgery. J Oral Maxillofac Surg 46:269-270

17. Dolan P, White RP Jr (1996) Community hospital charges for orthognathic surgery. Int $\mathbf{J}$ Adult Orthod Orthogn Surg 11: 253-257 